\title{
LHomme
}

L'HOMME Revue française d'anthropologie

206 | 2013

Varia

\section{Parenté sans sexualité}

Le paradigme occidental en question

Kinship without Sexuality. The Western Paradigm Called in Question

\section{Chantal Collard et Françoise Zonabend}

\section{(2) OpenEdition}

\section{Journals}

Édition électronique

URL : http://journals.openedition.org/lhomme/24516

DOI : 10.4000//homme.24516

ISSN : 1953-8103

Éditeur

Éditions de l'EHESS

\section{Édition imprimée}

Date de publication : 4 juin 2013

Pagination : 29-58

ISSN : 0439-4216

\section{Référence électronique}

Chantal Collard et Françoise Zonabend, «Parenté sans sexualité », L'Homme [En ligne], 206 | 2013, mis en ligne le 03 juin 2015, consulté le 19 avril 2019. URL : http://journals.openedition.org//homme/24516 ; DOl : 10.4000/lhomme.24516 


\section{Parenté sans sexualité \\ Le paradigme occidental en question}

\section{Chantal Collard \& Françoise Zonabend}

LES PROFONDS bouleversements intervenus en Occident dans la configuration du groupe familial et provoqués par l'augmentation des familles recomposées, adoptives, mono- ou homoparentales, s'accompagnant de la démultiplication des liens de parenté avec la mise en place de la procréation médicalement assistée (PMA), ont entraîné de multiples mutations dans nos modes de construction et, donc, de représentations de la famille et de la parenté.

La PMA tout particulièrement, qui ouvre sur une manipulation complexe des cellules embryonnaires pour aboutir, peut-être d'ici peu, aux procréations totalement artificielles à partir de cellules souches, a donné lieu à toutes sortes d'opérations procréatives : don de spermatozoïdes ou d'ovocytes, double don de gamètes, sélection et congélation d'embryons, prêt d'utérus, adoption d'embryons surnuméraires, implantation de gamètes et d'embryons post mortem. Les moyens sont multiples actuellement pour aider la nature à faire un petit d'homme. Si bien que l'on sait, maintenant, fabriquer des enfants hors sexe (par fécondation in vitro), hors corps d'une mère filiative (par l'intermédiaire d'une gestatrice) et même hors vie, puisqu'on peut devenir biologiquement père ou mère après sa mort (par prélèvement de gamètes ante mortem, voire même post mortem $)^{1}$. Aujourd'hui, un enfant peut donc avoir jusqu'à trois «mères »

1. En août 2002, en Israël, a été prélevé, puis congelé, à la demande de sa mère, le sperme d'un soldat mort au combat (Pullman 2010 : 14).

Cet article a fait l'objet d'une présentation en juin 2011 dans le cadre du séminaire de l'équipe "parenté» du Laboratoire d'anthropologie sociale. Nous remercions Klaus Hamberger, Laurent Barry et Michael Houseman pour leurs commentaires et suggestions après lecture de ce texte. 
- une d'intention, une donatrice, une gestatrice - et/ou deux "pères " l'un biologique, l'autre d'intention -, lesquels peuvent être morts ou vivants. Quant aux frères et sœurs "de gènes ${ }^{2}$ et autres demi-germains génétiques, ils peuvent être si nombreux qu'ils resteront à jamais des inconnus les uns des autres. Si l'on ajoute, en se rapportant aux paroles de René Frydman ${ }^{3}$, que demain on pourra sans doute concevoir des enfants sans désir, sans sexualité, sans origine, à partir de cellules souches, l'artificialisation de la conception et de la gestation devient de plus en plus réelle et donc réalisable.

Toutes ces «manœuvres" pratiquées de façon ordinaire dans le champ de la famille et de la parenté au long de ces trente dernières années imposent à l'anthropologue un impératif de compréhension. Au reste, celles-ci ont déjà suscité de nombreux travaux que nous tenterons de commenter plus loin. Nous voudrions ici apporter notre pierre à l'édifice en nous attachant à les analyser à partir du seul critère qui leur soit commun, au-delà des grandes différences qui existent entre elles. Toutes, en effet, engendrent de la parenté sans le recours à l'union sexuelle de l'homme (et) ou de la femme qui en sont les bénéficiaires. D'où le titre quelque peu provocateur de cet article! Pour autant, nous ne dénions absolument pas une activité sexuelle aux pères ou mères receveurs, qu'ils soient mariés, concubins, divorcés, pacsés ou célibataires, homo- ou hétérosexuels, ni même le fait qu'ils aient pu avec d'autres partenaires procréer des enfants par coït, mais, dans ce contexte-là, leur "sexualité est stérile ${ }^{4}$. Non plus que nous ne méconnaissions le fait que ces enfants

2. Comme le remarque Geneviève Delaisi de Parseval (2008), nous n'avons pas de terme pour désigner cette relation génétique qui existe entre des enfants issus du même don de gamètes ou d'embryons. Elle suggère d'utiliser en français le terme de sibling, mais cela ne convient pas pour les anglophones chez qui ce mot est déjà usuel. Nous ne pouvons pas non plus employer le terme de "germain " associé dans la nomenclature française à une relation de parenté vécue tant sociologiquement que biologiquement. Il s'agit, en fait, de nommer une relation de parenté naturelle assortie, le plus souvent, d'aucun lien de parenté socialement reconnu mais qui, néanmoins, ouvre sur des interdits sexuels et matrimoniaux: "frères et sœurs de gènes " nous semble une qualification possible.

3. "Demain, l'utérus artificiel fera-t-il le lien entre les 21 semaines qui séparent l'embryon in vitro de la couveuse du prématuré ? Mais, plus étonnant encore, il sera possible de créer artificiellement des gamètes, ces néogamètes, c'est-à-dire des spermatozoïdes et des ovules constitués artificiellement à partir des cellules-souches. Ces fameuses cellules à l'origine de toutes les autres, dès lors la conception in vitro par ces gamètes artificiels, puis le développement embryonnaire entièrement en laboratoire, aboutiraient à la production d'un être humain [...]. Le patrimoine génétique des gamètes proviendrait, certes, de cellules souches, mais cultivées de longs mois, voire des années, l'on aura oublié l'origine. Le passé ne se transmet pas dans les gènes mais dans les paroles d'un père et d'une mère qui, ici (à supposer que les enfants soient élevés par des humains normaux), ne sauraient quoi transmettre, sinon une mémoire" (Frydman 2008 : 223-224).

4. Pour reprendre, ici, le titre d'un des chapitres de l'ouvrage collectif de Claudia Mejia Quijano, Marc Germon et François Ansermet (2006: 205 sq.). 
adoptés, procréés in vitro ou enfantés par d'autres, soient issus du mélange de gamètes, mâles et femelles, encore nécessaires à toute conception humaine. De sorte que l'on a affaire à des personnes réceptrices de dons de gamètes, d'embryons, ou d'enfants adoptés qui vont constituer ces familles de types nouveaux ; toutes s'affirment "parentes" avec ces rejetons pourtant conçus entièrement ou partiellement par d'autres. En revendiquant ce titre, nous voulons insister sur la nécessité qu’il y a, pour l'anthropologue du moins, à "penser" ces nouvelles façons de procéder pour "faire famille» ou pour "entrer en parenté", à la même aune que ces parentés traditionnelles qu' elles soient biologiques ou sociales qui sont encore aujourd'hui, réellement ou symboliquement, aux principes mêmes de nos façons de vivre la parenté. Principes que ces nouvelles parentés tentent toutes de s'approprier. Cela nous conduit, dès lors, à revenir sur les différentes représentations à partir desquelles, en divers lieux et à des époques variées, on a institué et pensé les fondements de la filiation, et sur la place qu'y ont occupé la sexualité, le modèle procréatif ou d'autres socialement construits. Ce tableau brossé à grands traits nous fera comprendre l'intérêt qu'il y a d'ausculter dans toutes leurs dimensions ces nouvelles parentés en élargissant le débat, et de les discuter à partir des piliers conceptuels qui organisent classiquement l'anthropologie de la parenté : la filiation, l'alliance, la germanité, ainsi que le rôle structurant que joue la prohibition de l'inceste dans la constitution du groupe des Mêmes, ces Autres qu'on reconnaît comme semblables à Soi et que l'on nomme "parents» (Barry 2008). Il nous faudra, ensuite, tenter d'analyser ce qui s'accentue ou demeure encore vivant ou actuel, parmi ces grands axiomes que sont, en Occident, le principe généalogique, ainsi que le symbole-clé de la parenté euro-américaine sur lequel repose l'édifice culturel de notre système cognatique, l'union sexuelle de l'homme et de la femme (Schneider 1980 [1968]). Cela pour saisir, enfin, comment une sexualité et donc une corporéité (re)surgit néanmoins avec force au cours du processus de construction de ces nouveaux modes de la parenté occidentale, alors qu'elle était absente au départ.

\section{Aux fondements de la parenté}

Est-ce l'effet de la mode ou le fruit du hasard? Mais, dans le même temps où, dans nos sociétés contemporaines, se profilaient de nouvelles pratiques de procréation des enfants avec tiers donneur, où se multipliaient les façons d'entrer en parenté par adoption de tous types, et ce dans des configurations familiales traditionnelles ou insolites, des recherches anthropologiques et historiques portant sur la nature et les assises de la parenté étaient poursuivies tant dans les sociétés dites exotiques que dans 
le monde occidental passé ou actuel, à partir d'une relecture analytique et théorique approfondie de terrains ethnographiques ou historiques connus, ou par l'exploration de nouvelles sources de connaissances ${ }^{5}$. Ces recherches récentes ont approfondi l'analyse de ce champ d'études, montrant sa complexité, sa variabilité et la nécessité, pour mieux le circonscrire, de prendre en compte un ensemble de caractéristiques techniques, sociales ou symboliques, en sorte qu'aujourd'hui on sait qu'un large spectre de variations filiatives ordonne le champ parental et que la diversité est grande en ce qui concerne la reconnaissance accordée à la contribution de chacun (humains, ancêtres, déités, esprits ou substances vitales) à la venue au monde d'un enfant, à sa construction identitaire et à sa proximité avec certains parents au détriment d'autres.

Ainsi, nombre de sociétés, notamment patrilinéaires, ne peuvent laisser à la femme seule le soin de la conception, de la croissance et de l'engendrement de l'enfant. L'homme - père, mari, concubin ou amant - doit prendre sa part à toutes les étapes du processus procréatif, parfois même les effacer par des rituels appropriés, surtout lorsqu'il s'agit d'enfant de sexe masculin. Dans la Grèce d'Aristote, par exemple, l'homme et la femme détiennent une part fort différente dans la confection de l'embryon et dans sa croissance. La femme, avec le sang des règles, est là pour fournir la matière de base, mais l'homme par son mouvement et sa semence l'imprègne puis le forme. En quelque sorte le père, seul, incarne et transmet le modèle de l'espèce: l'homme engendre l'homme (Sissa 1991). Ailleurs, c'est la naissance elle-même qui n'est pas jugée suffisante pour faire "naître" au monde un enfant et les exemples de couvade - où le père se substitue, pour partie à la mère - sont nombreux dans la littérature anthropologique. Chez les Baruya de Nouvelle-Guinée, c'est le temps de la gestation qui est perçu comme incomplet pour faire des garçons qui sont engendrés deux fois, une fois par leur père et mère, une seconde fois, lors de l'initiation, par le groupe des jeunes hommes et avec l'aide du soleil (Godelier 2004). Il est certain que les sociétés à filiation matrilinéaire accordent plus d'attention ou de fonction aux attributs et substances maternelles dans le processus d'engendrement, mais pour autant, le masculin n'y est pas totalement absent. Ainsi, chez les Na matrilinéaires de la province du Yunnan en Chine, on pense que les fotus sont déposés par une divinité féminine dans le ventre des femmes qui les façonnent et

5. Cf., entre autres, les travaux de «revisite»: Annette Weiner pour les Trobriandais (1987), ou Sharon Hutchinson (1996) pour les Nuer. Citons parmi les textes généraux les plus récents sur la parenté : Viveiros de Castro (2004b); Godelier (2004); Barry (2008); Shapiro (2008); Héran (2009); Bamford \& Leach (2009); Porqueres i Gené, ed. (2009); Edwards \& Salazar (2009); Sahlins (2011); Bonte, Porqueres i Gené \& Wilgaux (2011); Carsten (2011). 
leur donnent os et chair, et donc qu'ils préexistent aux relations sexuelles et ne proviennent pas d'elles. L'homme, qui certes n'est "ni père ni mari », joue néanmoins un rôle puisque, par son sperme appelé l' " eau de pénis ", il fait croître ces foutus comme la pluie fait pousser l'herbe! (Cai 1997).

Par ailleurs, de nombreuses ethnies n'ont pas pris pour acquis que la fécondation par copulation constituait le moment fort, voire exclusif, de la transmission des substances corporelles et, par là, des ressemblances, comme nous l'avons posé dans notre modèle occidental. Ainsi les Trobriandais matrilinéaires de Nouvelle-Guinée affirment-ils que le mari de la mère ne joue aucun rôle dans la conception de l'enfant, mais ceux-ci déclarent tout également que les enfants lui ressemblent autant qu'ils ressemblent aux membres de son matrilignage, et qu'il n'existe aucune similitude entre ses rejetons et leur mère - c'est même une grave offense que d'y faire allusion. Selon l'explication indigène, cette ressemblance tient au fait que le mari de la mère modèle, avec son pénis, le visage de l'enfant en ayant des relations sexuelles répétées avec elle pendant la gestation (Malinowski 1929; Weiner 1987). Au demeurant, dans d'autres modèles procréatifs, la transmission de substances, tant masculines que féminines, peut avoir lieu lors de la fécondation comme nous l'avons noté dans la Grèce antique ou pendant la gestation. Chez les Txicào du Brésil (Menget 1988), on estime même que le nourrissage du foetus doit être une tâche collective des hommes du proche entourage, qui, par leurs relations sexuelles répétées avec la mère, vont contribuer à faire fructifier l'enfant à venir. Ici, la paternité ordinaire ou solitaire ne suffit pas à la bonne fabrication d'un enfant. Souvent aussi, l'influence de substances vitales intervient après la naissance comme dans les sociétés arabo-musulmanes où l'on accorde une grande importance à la parenté créée par le biais de la lactation (Barry 2008 ; Fortier 2001). De même, chez les Jivaro Achuar (Taylor 2000), ni la copulation ni l'apport de sperme pendant la gestation ne suffisent à nouer le lien de paternité. Certes, le géniteur doit "nourrir » l'enfant pendant la grossesse, mais surtout il doit agir comme père nourricier pendant la petite enfance s'il veut acquérir le statut de parent.

En soulignant d'autres lectures possibles des faits de nature dans la fabrication des humains et en insistant aussi sur la temporalité associée à la croissance du bébé avant et après la naissance, ces travaux ethnologiques ont ainsi conforté les nouvelles formes de paternité et de maternité émergentes en Occident. En outre, en approfondissant l'étude dans certaines sociétés de la germanité, en insistant sur la place originale et originaire que tient ce lien de parenté, elles ont permis une autre compréhension et appréhension de cette relation familiale dont ces nouvelles parentés occidentales sont, nous le verrons plus loin, fortement tributaires. 
Dans certains groupes sociaux, l'utérus, le ventre féminin, est considéré comme le lieu et le lien essentiels dans l'instauration d'une relation commune de germanité entre enfants engendrés par une même mère. C'est notamment le cas des Malays de Langawi, où le placenta est perçu comme un germain de l'enfant en gestation. Après la naissance, celui-ci est d'ailleurs enterré comme le serait un enfant mort-né (Carsten 1995). La même situation se retrouve chez les Kamea de Nouvelle-Guinée où tous les enfants qu'une femme a portés, quel que soit leur géniteur, sont dits «être du même sang " ou plus exactement d'un "seul sang ", alors que les demi-germains paternels eux ne sont jamais one-blood (Bamford 2007), tout comme la mère n'est jamais dite one-blood avec ses enfants, même si l'on reconnait que, comme le père, elle transmet des substances corporelles à ses enfants, substances auxquelles d'ailleurs on n'accorde que peu d'importance dans la formation du lien intergénérationnel ${ }^{6}$. Tout se passe comme si le fait de partager le même abri, la même "maison » renforçait, entre les occupants, leur appartenance parentale. Dès lors, c'est la relation de germanité, donc la collatéralité, qui est au fondement de la parenté, et non les liens ascendants.

On connaît aussi, de par le monde, quelques cas rares de sociétés où c'est la sexualité elle-même qui ne semble pas être particulièrement prisée, le groupe social préférant d'autres modes d' "entrée en parenté " pour renouveler ses membres et perpétuer les lignées. Dès lors, la construction sociale de liens filiaux se révèle être plus appréciée que celle issue de la parenté naturelle, les relations de parentalité électives étant considérées comme plus propices à la mise en place d'une véritable parenté. Il en est ainsi chez les Mbaya-Guaicuru du Brésil, étudiés par Claude Lévi-Strauss (1976 [1955]), où l'on adopte les enfants des ennemis tués au combat, ou chez les Baining de Nouvelle-Bretagne qui s'échangent entre eux leurs enfants :

"Chez les Baining, ces derniers [ceux qu'on a conçus] suscitent la honte de leurs géniteurs, la sexualité étant dévalorisée. Les termes de parenté (père/mère/fils/fille) ne sont utilisés qu'entre parents et enfants adoptifs. [...] [L]'adoption se fonde sur le don et l'échange qui sont au cœur du lien social. Comme si la filiation devait être amputée de ses bases biologiques et reconstruite par le nourrissage à l'aide d'aliments issus du labeur " (Juillerat 2005: 86).

6. "While both a mother and a father are understood to contribute bodily substance to the making of a child, this is seen to be a rather unremarkable (and certainly an unremarked upon) feature of the ensuing relationship. My own efforts to ground motherhood and fatherhood in some kind of substance-based link met with everything from amusement to total indifference and incomprehension" (Bamford 2007 : 302). 
Mais, il n'est nul besoin d'éprouver une telle répulsion envers la procréation pour faire appel à d'autres modes d'entrée en parenté sans sexualité. Que ce soit en Occident ou ailleurs, il est assez banal de rencontrer, dans une même société, plusieurs façons de se fabriquer des parents: l'une avec sexualité procréative, les autres sans. Très souvent, d'ailleurs, ces parentés acquises sans sexualité copient la parenté naturelle ou sont en continuité avec elle par certains aspects. Dans nos sociétés chrétiennes, par exemple, le rituel du baptême fait (re)naître l'enfant dans la communauté des croyants, lui donnant des "parents spirituels» véritables substituts de père et de mère qui l'accompagneront au long de sa vie, en créant, en quelque sorte, entre les uns et l'autre un lien de quasi-filiation assorti souvent d'interdits matrimoniaux (Fine 1994; D’Onofrio 2004). En somme, pour l'église chrétienne et pendant longtemps dans les sociétés civiles d'Occident, l'acte de baptême a fait office d'acte de naissance.

À côté de la parenté naturelle issue de la procréation, l'adoption - celle qui implique un changement de filiation - constitue certainement la pratique la plus usitée et recherchée dans de nombreuses sociétés anciennes ou contemporaines, exotiques ou européennes. Là encore d'importants travaux d'historiens (Corbier 1999) ou d'anthropologues (Leblic 2004 ; Fine \& Neirinck 2000 ; Héritier \& Copet-Rougier 1995) ont dressé le catalogue détaillé diachronique et géographique de cette institution, soulignant sa variabilité, sa complexité selon l'époque ou les cultures en cause, insistant sur le fait que celle-ci était assortie de modalités particulières adaptées au modèle culturel du groupe social.

Ainsi, il peut y avoir une grande ou, au contraire, une imperceptible différence entre parents adoptifs ou biologiques, comme chez les Malays Langawi dont il a été question plus haut, où le sang transmis par les géniteurs à leur enfant est constamment renouvelé par la nourriture qu’il mange, ce qui fait qu'un enfant adopté acquiert à la longue le même sang que sa famille adoptive (Carsten 1995). Exit, dans cet exemple, l'opposition traditionnelle qu'on a pu faire dans les études parentales, entre parenté biologique et parenté sociale. C'est pourquoi, pour mieux se distancer du concept de parenté classique, Janet Carsten propose d'utiliser le terme de relatedness ${ }^{7}$, que l'on peut traduire en français par "apparentement",

7. "The authors in this volume used the term "relatedness" in opposition to, or alongside, "kinship" to signal an openness to indigenous idioms of being related rather than a reliance on pre-given definitions or previous versions. In this introduction I have also used "relatedness" in a more specific way in order to suspend a particular set of assumptions about what is entailed by the terms social and biological. I use "relatedness" to convey, however unsatisfactorily, a move away from a pre-given analytic opposition between the biological and the social on which much anthropological study of kinship has rested" (Carsten $2000: 4)$. 
où s'entend bien l'action de mise en relation, de transmission d'une personne à l'autre jusqu'à ne plus former qu'une seule et même nature.

Dans une même perspective constructiviste, mais allant au-delà de cet auteur, se situent, nous semble-t-il, les récentes réflexions de Marshall Sahlins (2011). Ce dernier insiste sur le fait que les relations de parenté peuvent tout aussi bien être construites après la naissance, voire même longtemps après, en effectuant envers les personnes choisies les actions culturelles appropriées. Les sources les plus courantes de ces formes construites de parenté - elles suivent des logiques associatives particulières de mises en relation - sont l'adoption, la commensalité, le partage de nourriture, la réincarnation, la co-résidence, les mémoires ou souffrances partagées, le travail en commun, voire même l'amitié ${ }^{8}$; ou encore, la relation créée entre le tueur et sa victime par le sang versé. Prenant en compte ce catalogue aux entrées multiples, Sahlins en déduit que la relation de parenté consiste en la pratique d'un "échange mutuel entre les êtres " qui participent de façon intrinsèque à l'existence des uns et des autres, tout en insistant sur le fait que ces relations, la plupart du temps positives et où règne l'entraide, ne sont pas pour autant dénuées de jalousie, d'ambivalence, voire parfois de violence :

«Mutuality of being: persons who are members of one another, who participate intrinsically in each other's existence. "Mutuality of being" applies as well to the constitution of kinship by social construction as by procreation, even as it accounts for "the mysterious effectiveness of relationality" [Viveiros de Castro 2009], how it is that relatives live each other's lives and die each other's deaths" (Sahlins 2011 : 2).

Pour Sahlins, ainsi que pour Viveiros de Castro, les relations de parenté participent du même régime - positif ou négatif - et régissent tous les types de relations humaines, comme par exemple celles engendrées par la magie ou l'exercice du don (Ibid. : 237 sq.). Dès lors, on peut se poser la question de savoir quelle place occupe la parenté au sein de cet ensemble d'intersubjectivité relationnel? Pourquoi Sahlins lui donne-t-elle une position fondamentale au sein de cet ensemble? Cela reste pour nous inexpliqué! Selon notre auteur, en effet, nul besoin de distinguer ces relations les unes des autres, alors que pour notre part il nous semble que la parenté, de quelque façon qu'elle s'enclenche, s'inscrit différemment des autres pour autant que celle-ci, une fois enclenchée, a des effets

8. «A catalogue of commonplace post-natal means of kinship formation would thus include: commensality, sharing food, reincarnation, co-residence, shared memories, working together, adoption, friendship, shared suffering, and so on. But the performative modes of kinship known to anthropology - if not to socio-biology or evolutionary psychology - are indefinitely many, inasmuch as they are predicated on particular cultural logics of relatedness " (Sahlins 2011:4). 
indéniables, incontournables, sur le corps et les relations sexuelles des uns et des autres parents. Sahlins semble bien avoir traité de la parenté dans l'optique classique britannique de la filiation et de la descendance, sans vraiment tenir compte de la place de l'alliance et donc de l'inceste qui fondent, au même titre que la filiation et la germanité, la parenté 9 . Force est, aussi, de constater qu'en voulant mettre un bémol sur le modèle procréatif, Sahlins semble bien être passé d'un même mouvement pardessus le corps et les substances vitales, insistant d'abord sur les relations non charnelles voire même sur l'idée que la parenté consiste en une relation subjective, quasi émotionnelle en quelque sorte. Or, avec d'autres analystes de la parenté, nous nous posons la question de savoir si l'on peut se passer totalement de l'idiome du corps et du circuit des substances corporelles (Carsten 2011; Porqueres i Gené 2009) pour parler de parenté. Encore une fois, celle-ci ne se réduit pas à la seule filiation, mais compte aussi et surtout avec l'alliance qu'encadre la loi de l'inceste. C'est cette dernière qui dit, partout et toujours, quels corps peuvent se mêler définissant ainsi les frontières irréductibles de la parenté. Certes le nourrissage, la jouissance d'espaces ou de biens communs, la possession de mêmes ancêtres et d'autres traits encore, peuvent engendrer des formes d'apparentement. Mais ce n'est que lorsque ces aspects de partages entraînent ou imposent une interdiction de relation sexuelle et d'alliance, que prend forme «la » parenté à partir de laquelle celle-ci s'ordonnance et se hiérarchise pour autant que tous les apparentés ne sont pas tous interdits.

De même, dans les approches analytiques et théoriques de la parenté, il semble que l'on soit allé trop loin dans l'usage de la parenté classificatoire, comme le souligne Warren Shapiro $(2008,2012)$, auquel nous reprochons néanmoins d'être resté de façon trop étroite dans le modèle procréatif et généalogique ${ }^{10}$. Au prétexte que dans les sociétés exotiques, aussi bien que dans les nôtres, on désigne par un même terme de parenté plusieurs individus, on en déduit que ceux-ci sont tous identiquement parents. Avec cette façon de penser la parenté, on oublie un peu vite la "focalité ", c'est-à-dire le « cœur » irréductible de la hiérarchie de la parenté : parmi les individus consanguins ou alliés dénommés par un même terme, certains peuvent être perçus comme plus proches parents entre eux que d'autres,

9. En anglais le terme kinship a deux sens : la consanguinité et la parenté.

10. "Thus McKinnon [2005: 109-113] Sahlins' protégé, insists upon "a multiplicity of mothers" in the systems of kin classification Morgan called "classificatory", utterly oblivious to notions of modelling in anthropology or cognitive psychology [MacLaury 1991], as well as to the logical relationship, in English, between mother, on the one hand, and godmother, mother-in-law, and grandmother, on the other" (Shapiro 2008: 140-144). Sahlins ne commet pas cette erreur et reconnait la focalité : "Broadly speaking, mutuality of being among kinfolk declines in proportion to spatially and/or genealocally reckoned distance» (Sahlins $2011: 234$ ). 
car leurs relations s'inscrivent dans une corporéité et/ou une spatialité plus intime auxquelles certains qualificatifs font du reste référence. Ces gens-là sont nos vrais "parents" entre lesquels les assises fondamentales de la parenté - filiation, alliance, germanité, inceste - trament leurs réseaux.

\section{De la parenté dans un monde occidentalisé}

Avec la montée de l'individualisme, la démultiplication des acteurs parentaux ou de la recomposition des familles, on assiste en Occident à une diversification des configurations familiales (Martial 2003) et parentales telles les familles homoparentales (Cadoret 2009), qui toutes réclament haut et fort leur légitimité. La variété et la divergence des réponses sur ces questions, fournies par les législations nationales, fédérales ou provinciales dans des pays voisins géographiquement ou culturellement qui tous, pourtant, s'inscrivent dans le même système de parenté de type cognatique, montrent la grande flexibilité de celui-ci ${ }^{11}$. Ces réglementations sont en perpétuelle évolution sous la pression de la société civile ou sous l'effet de la puissance juridique qui, parfois, autorise des pratiques interdites par la loi nationale. Ainsi en France, la justice, en 2010 et en 2011, a homologué, par trois fois, des adoptions réalisées à l'étranger par des parents de même sexe ${ }^{12}$ au nom de l'intérêt de l'enfant, alors que cette pratique leur est interdite par la loi. De même, en ce qui concerne la reconnaissance de familles atypiques - comportant plus de deux parents -, signalons que de récents jugements de la Cour d'appel de l'Ontario (Canada) ont validé l'existence de trois parents dans le cas de couples de mères lesbiennes, arguant du fait que le donneur de sperme était connu et avait agi depuis la naissance de l'enfant en tant que père social ${ }^{13}$. On conçoit que ces décisions, qui ne sont que des cas particuliers, n'enclenchent pas immédiatement un changement de la loi, et reconnaissons cependant que le modèle généalogique s'en trouve quelque peu complexifié. Ces législations changeantes ou les décisions juridiques récentes relancent sans cesse les débats nationaux de société (Théry 2010) qui, toujours, demandent à être interprétés à la lumière de l'histoire et des mentalités particulières à chaque pays en tenant compte de leurs traditions

11. Cf., sur ce sujet, le tableau récapitulatif des législations en vigueur de par le monde, établi par Geneviève Delaisi de Parseval (2008 : 378-385).

12. Arrêt de la Cour de cassation du 8 juillet 2010, de la Cour d'appel de Paris du 26 octobre 2011 et de Bayonne du 26 octobre 2011. Cf. aussi l'article d'Anne Chemin, Le Monde du 26 mars 2011.

13. Le premier cas date de 2007 et a été jugé par la cour d'appel de l'Ontario (http://www.can lii.org/en/on/onca/doc/2007/2007onca2/2007onca2.html). Depuis, quatre jugements identiques auraient été rendus en Ontario. 
légales, de leurs croyances morales et religieuses. Mais, si ce qu'on ne peut faire chez soi est accessible dans les pays voisins ou lointains, on conçoit que le choix, dans un État, d'une législation plutôt qu'une autre, ait donné lieu à des débordements outre frontière ${ }^{14}$ et à un tourisme procréatif très actif, que les chercheurs qualifient de "délocalisation procréative ", mais que de nombreux parents d'intention, au vu des difficultés qu'ils ont rencontrées dans leur propre pays, qualifient plutôt d' " exil procréatif " ou, pour les adoptants homosexuels, d' " exil juridique ", soulignant par l'usage de ces descriptifs tout à la fois la souffrance subie et leur absence de choix.

Face à la prolifération de nouvelles configurations familiales et de modes pluriels parentaux possibles, faut-il pour autant en conclure que les fondements de la parenté ont été complètement bousculés en Occident et qu'il n'y a plus de repères? À y regarder de près, on constate certes qu'il y a eu des changements sociaux, mais ceux-ci sont dus d'abord à des ajustements nécessités par les découvertes scientifiques récentes et que cela est en continuité avec l'idéologie occidentale de la parenté (Schneider 1980 [1968]; Strathern 1995). Parmi les percées scientifiques majeures qui ont bouleversé le paysage familial et parental, viennent immédiatement à l'esprit celles ayant trait à l'ADN, lesquelles permettent désormais de déterminer avec certitude une paternité (jusque-là toujours incertaine) et ont perturbé nos façons de penser la filiation (Brunet 2011) ; puis, celles conduisant à la congélation des gamètes et des embryons et pouvant ouvrir sur un "brouillage » des générations, mais aussi sur la possibilité de la gestation pour autrui, si bien que la maternité biologique est désormais divisible; enfin, celles qui débouchent sur une meilleure connaissance des mécanismes génétiques de la transmission héréditaire et permettent aujourd'hui, entre autres possibilités, de pouvoir choisir, après un double diagnostic génétique et préimplantatoire, le sexe souhaité ou l'enfant compatible avec des visées thérapeutiques. Les découvertes scientifiques sur les gènes ont eu un tel impact sur nos représentations et pratiques de la parenté que des chercheurs en ont conclu que l'on assistait, dans ce contexte, à une "génétisation" de la parenté en Occident (Finkler 2001). Il nous semble, pour notre part, qu'il serait plus juste de dire qu'on observe un glissement, dans les représentations de la parenté naturelle, de la consubstantialité ou encore du sang, vers les gènes (Delaisi de Parseval \& Collard 2007 ; Porqueres i Gené 2009) ; d'un autre côté, comme l'ont fait remarquer plusieurs chercheurs, ces gènes continuent aussi d'être très sociaux (Edwards 2009; Bestard 2009).

14. Sans parler des trafics d'enfants ou de marchandisation des substances qui ont lieu, aujourd'hui, par l'intermédiaire d'internet. 
En outre, la multiplication des figures parentales, tant biologiques que sociales, a peu à peu complexifié le modèle généalogique. Ainsi, dans les cas de PMA ou d'adoption au bénéfice des couples homosexuels ou hétérosexuels, un acte de naissance est dressé au nom des deux parents d'intention - qui, aujourd'hui, peuvent être de même sexe dans nombre de pays -, mais ces écritures ne gomment pas pour autant les cogéniteurs ou les parents d'origine. Les nombreux discours sur l'importance, pour l'enfant, de connaître ses origines génétiques et son histoire biographique sont là pour le prouver.

Toutefois, comme l'ont souligné certains travaux, la parenté sociale, créée par adoption ou recomposition familiale s'affirme elle aussi haut et fort (Fine \& Martial 2010). En sorte qu'aujourd'hui on y insiste souvent avec une grande vigueur : la "chair» à elle seule ne suffit jamais à "faire famille " et la "volonté " de devenir parent et le "temps" sont fondamentaux dans le processus « de familialisation ou d'emparentement » (kinning: cf. Howell 2003; Zonabend 2007). En fait, il faut toujours, d'une façon ou d'une autre, adopter ses propres enfants et décider d'agir auprès d'eux en tant que parent. Nous en prendrons pour preuve les dires de ces femmes qui se préparent à avorter et qui toutes décrivent l'embryon qu'elles portent non pas comme un enfant mais plutôt comme une tumeur (Boltanski 2004), ou encore ces naissances monstrueuses, parce que non voulues, imaginées par la science-fiction (Moisseeff 2002). Ces solides arguments d'une volonté filiative et d'une vie de proximité sont également ceux revendiqués par les parents d'intention ou par des adoptants. Toutes ces constatations nous amènent à conclure, avec Jeanette Edwards (2009), que si, d'un côté, les mécanismes scientifiques de la parenté biologique sont de mieux en mieux connus et explorés, de l'autre, le discours sur la parenté sociale est lui aussi de plus en plus explicite, revendiqué et affirmé. Au demeurant, avec la PMA, peut-on encore parler de parenté " naturelle » quand les manipulations de l'engendrement sont aussi complexes et diversifiées? Aussi, aujourd'hui, il est courant d'accoler le vocable "hybride» à ces parentés dans lesquelles les deux domaines, nature et culture, sont tout à la fois complètement remaniés et indéfectiblement liés (Strathern 1992 ; Thompson 2005).

Toutes ces études néanmoins, avec ce qu'elles ont pu apporter de positif, ont mis l'accent sur le «type » de parenté et sur la construction de liens de parentalité particuliers dans ces nouvelles familles. Ce faisant, on a quelque peu perdu de vue les autres assises classiques de la parenté : filiation, germanité, alliance et, tout particulièrement dans cette parenté sans sexualité, le rôle que joue la prohibition de l'inceste dans la consolidation du " groupe des Nous », " des Mêmes " par opposition aux " Autres », aux non-parents. 


\section{L'universel de l'inceste}

"À l'heure où nos sociétés postmodernes ne voient plus dans l'homosexualité un "problème" mais bien plutôt un choix, il n'est plus des grands interdits sociaux que celui de l'inceste qui soit toujours tenu pour parfaitement légitime par l'immense majorité d'entre nous sans pourtant que nous ayons jamais été à même de justifier ni de l'origine ni du sens d'une telle prohibition» (Barry 2013).

Malgré des changements considérables intervenus dans le champ de la parenté et de la famille, la prohibition de l'inceste et la loi d'exogamie qu'elle sous-tend continuent toujours à s'appliquer, comme le remarque cet auteur, et cette permanence devrait nous faire réfléchir de même que les transformations que ce champ subit. Ces prohibitions sexuelles et empêchements de mariage sont d'autant plus intéressants à étudier, qu'avec la règle des adoptions sans précision dans les dossiers de la mère et/ou de la famille de naissance, ou des dons anonymes en PMA qui ont cours dans de nombreux pays occidentaux, on assiste depuis une trentaine d'années à la reviviscence d'un vieux cauchemar qui de tout temps a hanté nos sociétés ${ }^{15}$ : celui de l'inceste involontaire ou encore appelé "accidentel ${ }^{16}$. Il faut mettre de l'ordre dans le grand chaos que ces parentés non régulées ont engendré. Or, s'il y a eu un regain d'intérêt, en France notamment, pour tenter de proposer une théorie unitaire de l'inceste ${ }^{17}$, fort peu de travaux à ce jour s'attachent à analyser les pratiques et les représentations sociales de celui-ci en Occident actuellement.

Autrefois, les anthropologues avaient jugé nécessaire dans leurs approches théoriques de distinguer les relations sexuelles du mariage. Or, comme le note Jeanette Edwards (2004) ou Corinne Fortier (2005), depuis l'apparition des nouvelles technologies de fabrication d'un humain, il faut, de plus, dans l'étude du phénomène, isoler la procréation des relations sexuelles et du mariage et, dès lors, élargir le champ d'application

15. «Au haut Moyen-Âge et même avant, de nombreux auteurs se sont insurgés contre le fait d'abandonner ou d'exposer les enfants non désirés, pour autant que ceux-ci pouvaient survivre et commettre, plus tard par ignorance, un inceste avec un membre de leur parenté » (Boswell 1993: 270 et note p. 357).

16. Comme l'écrit Naomi Cahn : «Incest is a significant concern in the new world of test tube families. In 1980, the geneticist Martin Curie-Cohen raised the possibility of "inadvertent inbreeding" from the increasing use of donor sperm. Others have called this "accidental incest," in which "the offspring of donated sperm or ova meet and are unknowingly attracted". The fear is pervasive in the reproductive technology world" (2009: 59-60).

17. Les auteurs en particulier ont porté attention aux logiques symboliques complexes tournant autour du corps, associées à la prohibition. Ils ont aussi proposé de nouvelles théories et en ont débattu entre eux. Parmi les nombreux travaux, signalons en tout premier lieu ceux de Françoise Héritier (1994) sur l'inceste du deuxième type, mais aussi ceux de Salvatore D'Onofrio (2004) sur l'inceste spirituel du "troisième type " dans la chrétienté, de Bernard Vernier sur la théorie de l'inceste en regard de l'ethnographie grecque (2009). 
de l'inceste pour y inclure une nouvelle modalité qui s’instaure avec la parenté sans sexualité - mais pas sans substances - issue de dons de gamètes ou d'embryons et de la gestation pour autrui.

Le champ d'étude de l'objet ainsi élargi, de quelle manière l'aborder?

On pourrait certes en débattre d'un point de vue psychanalytique pour autant que ces nouvelles réalités parentales introduites dans nos sociétés occidentales par des manipulations hors sexe et hors corps « correspondent à un parfait désaveu de la scène primitive qui pose à l'origine du bébé l'union de deux corps de sexe différent" (Mejia Quijano, Germond \& Ansermet 2006: 210). Nous ne saurions reprendre ici leurs nombreux travaux sur les conséquences psychologiques individuelles ou sociétales de ces situations. Nous retiendrons, toutefois, de ces explorations psychiques, la place prépondérante qu'elles donnent au savoir réel ou imaginaire, sur l'«origine » dans la faisabilité d'une relation de parenté ou de parentalité entre parents et enfant.

Surtout, ces réflexions psychanalytiques ne suffisent pas à cerner l'ensemble des répercussions filiatives, sociales et morales très particulières engendrées par ces nouvelles façons de se fabriquer de la parenté. Il est indispensable d'y adjoindre, à notre sens, une approche ethnographique fine des pratiques quotidiennes, des activités pragmatiques et des discours qui, dans ces nouvelles familles, y sont associés, voire même comme l'a fait en Grande-Bretagne, mais avec très peu d'informateurs, Jeanette Edwards (2004), de recueillir l'opinion du grand public sur des situations hypothétiques de dons de gamètes intrafamiliaux ou de gestation pour autrui. La parenté est en effet une affaire qui nous concerne tous et autour de laquelle il faut que s'élabore un savoir partagé. Jusqu’à présent ce volet ethnographique est peu étoffé.

L'analyse des législations nationales ou provinciales et celle des recommandations réglementaires associatives ou institutionnelles, qui sont les deux modes d'organisation du domaine de la procréation sans sexualité dans les pays occidentaux, nous donnent néanmoins déjà quelques indications ${ }^{18}$. Une première constatation s'impose : dans tous les pays qui autorisent de telles manipulations, on cherche à aligner les prohibitions des dons de gamètes sur les interdits incestueux impliquant des consanguins de «sexe différent ». Les protocoles des cliniques de fertilité ou les lois de bioéthiques interdisent donc tous les dons directs de sperme de père à fille, de fils à mère ou de frère à sœur, ainsi que les dons d'ovocytes d'une sœur à son frère ou d'une mère à son fils et d'une fille à son père.

18. Aux États-Unis, la PMA ne fait pas l'objet d'une législation nationale, mais reste du ressort des différents États et institutions médicales. Il est alors nécessaire et instructif de consulter les règlements internes des cliniques de fertilité, ainsi que les recommandations de leurs comités de bioéthique. 
Pour les dons de gamètes entre germains ou parents et enfants de "même sexe ", on note une grande disparité entre les dons de sperme d'un homme à un autre (ou à un couple) et les dons d'ovocytes d'une femme à une autre (ou à un couple). Cette différence avait déjà été notée par Charis Thompson (2005) en ce qui concerne les États-Unis, où $60 \%$ des cliniques de fertilité répertoriées autorisaient les dons de sperme de frère à frère, alors que $90 \%$ de ces mêmes cliniques acceptaient les dons d'ovocytes de sœur à sœur. Devant cette disparité, il est intéressant de noter à ce sujet qu'un comité d'étude sur l'éthique et le droit de la société européenne de reproduction humaine et d'embryologie (EsHre Task Force on Ethics and Law) a recommandé, en 2011, qu'au nom de la justice, des cas similaires soient traités de la même façon et que, si l'on accepte les dons d'ovocytes de sœur à sœur, il serait arbitraire de ne pas autoriser les dons de sperme de frère à frère, à moins, ajoute-t-elle, qu'il existe une différence significative au niveau moral, entre ces types de dons intrafamiliaux. Or, l'écart relevé par Thompson s'observe dans plusieurs pays, dont le Canada, mais concerne aussi plusieurs États d'Europe.

D'après les enquêtes de Chantal Collard auprès des cliniques de fertilité de l'Ouest et du Centre canadien, les dons de sperme de frère à frère sont quasi inexistants, alors que les dons d'ovocytes de sœur à sœur, sans être majoritaires, sont plus courants ${ }^{19}$. Au Canada, la loi sur la procréation assistée (2004) interdit toute rétribution pour dons de gamètes et autorise les dons directs entre donneurs proches et connus. Or, il faut savoir que les dons de sperme de tous donneurs doivent être testés, pour éliminer la possibilité de maladies sexuellement transmises et que, pour ce faire, le sperme frais est analysé, puis cryoconservé pendant six mois, puis analysé de nouveau ${ }^{20}$. Le protocole médical prend du temps et interdit tout transfert fécond immédiat. Ces procédures tant techniques que financières expliquent, en partie, pourquoi, dans les consultations médicales, on n'aborde jamais la possibilité de dons directs de frère à frère. Cela est différent dans les cas de dons d'ovocytes, où le transfert entre donneuse et receveuse peut être exécuté immédiatement, aussi recommande-t-on aux couples de chercher une donneuse parmi leurs sœurs et amies. Remarquons cependant que ce don est loin d'être un acte anodin. Non seulement il prend aussi du temps mais suppose, de plus, de la part de la donneuse d'accepter d'être astreinte à de longs examens biologiques, cliniques et psychologiques, puis d'être soumise à une stimulation ovarienne

19. Ainsi, une clinique de Vancouver estimait que 17 des 35 cas de dons directs effectués dans ses murs impliquaient des sœurs.

20. Informations fournies par Shireen Kashmeri (enquête en cours). 
contraignante et une ponction sous anesthésie générale. On peut comprendre, au vu de ce lourd protocole médical, pourquoi dans de nombreux pays on manque cruellement d'ovules ${ }^{21}$. Cependant, les conditions économiques et techniques n'expliquent absolument pas ce différentiel entre dons d'ovules et de sperme. Il faut plutôt pour le comprendre, croyons-nous, se tourner vers les représentations communément partagées dont sont toujours tributaires, dans nos sociétés, la sexualité et l'inceste, et force est de reconnaître alors que sperme et ovule ne sont pas pensés comme équivalents.

Déjà, Aristote, dans le Traité de la génération des animaux (cf. 2002), posait que la substance masculine était active, intrusive, pénétrante, au contraire de la substance féminine qui, elle, était vue avant tout comme passive et réceptrice. Du reste, de nos jours l'inceste, dans les représentations sociales ou descriptions légales, est encore perçu et caractérisé comme un acte impliquant une pénétration, une intromission violente. Dans cette optique, on conçoit dès lors que l'échange de substance entre frères - qui implique toujours le corps d'une femme ${ }^{22}$ - peut être perçu comme plus incestueux que les dons directs entre sœurs. S'amalgame à ces représentations différenciées le stéréotype de la femme vue avant tout comme "altruiste", une perception à laquelle s'adjoint le fait avéré, incontestable jusqu'à présent, que celle-ci représente le seul " truchement » capable d'enfanter et de perpétuer une lignée. Pour elle, le «don d'engendrement " (Théry 2010) est pensé comme normal et allant de soi. Tous ces arguments concourent à effacer, voir à brouiller tout soupçon de relation incestueuse entre femmes. Notons, enfin, que la paternité biologique n'est pas divisible contrairement à la maternité qui peut se séparer entre le génétique et le gestationnel : la receveuse d'ovocytes portera, nourrira et accouchera de l'enfant, c'est dire que viendront s'ajouter à l'embryon qu'elle a reçu d'autres substances féminines qui rendront celui-ci moins étranger à la gestatrice.

21. Cette situation a entraîné, dans de nombreux pays, un véritable "marché noir " des ovocytes. Cf. Beijing Journal News, "A Child at Any Cost : Beijing's Black Market of Student "Egg Donors" Exposed ", article du 30 novembre 2011 (http://www.echinacities.com/china-media/A-Child-atAny-Cost-Beijings-Black-Market-of-Student-Egg-Donors-Exposed). En France, au tout début de la pratique de la PMA, les dons d'ovules entre sœurs ou proches parentes étaient acceptés dans quelques centres hospitaliers. Actuellement, la loi de bioéthique française de 1994 a mis fin à cette possibilité. On peut certes venir pour une PMA avec une donneuse, toutefois celle-ci ne donnera pas ses "œufs » au couple recruteur, mais à un autre qu'elle ne connaît pas et qui ne la connaît pas. Mais, le fait de recruter une donneuse permet de raccourcir le délai d'attente.

22. "In the examples presented here, the notion of incest is evoked in the inappropriate mingling of body substances that ought not to mingle. This does not depend on sexual intercourse, but it does require the bodies of women, even if the initial mingling is in a petri dish " (Edwards $2004: 770)$. 
Ni les biologistes ni les médecins n’ont pleinement saisi les différences sociales et symboliques qui perdurent, en ce qui concerne les perceptions de l'inceste, entre les deux types de dons. En réactivant à travers leurs pratiques - ordonnancées sans y attacher trop de poids - un biais patrilinéaire, ces nouvelles formes de procréation confirment, s'il en était besoin, que l'élaboration de la parenté se poursuit même en laboratoire: se trouve ici renforcée une vision spermatique masculine de la procréation.

Dans les règlements des cliniques comme dans les législations, un débat, tout aussi sensible s'instaure autour des dons intergénérationnels et intrafamiliaux, qu'on ne retrouve là encore souvent qu'entre femmes: mère/fille ou fille à mère, ou encore nièce à tante ou tante à nièce, pour autant qu'ils posent avec acuité la question de l'autonomie des personnes en cause ${ }^{23}$. Charis Thompson (2005) évoque le cas d'une femme infertile qui devient mère grâce à un embryon conçu avec l'ovocyte de sa fille née d'un premier mariage et le sperme de son actuel époux, porté par une mère gestationnelle. À Montréal, au McGill Reproductive Centre, a été prise une décision considérée comme une première mondiale : une femme a été autorisée à congeler ses ovocytes pour l'usage futur de sa fille née avec une maladie génétique qui la rendra stérile, mais qui lui permettra tout de même de porter un enfant. Quel peut être, dans ce tissu d'intimité qui lie mère et fille, la liberté de décision de l'une ou de l'autre? De plus, ne peuton soupçonner dans ces cas une situation incestueuse relevant, en quelque sorte, d'un abus d'autorité entre enfants et parents ou entre aînés et cadets?

Si les dons au plus proche peuvent conforter l'identité, la cohésion du groupe des mêmes au niveau de la chair, le principe de l'ordre généalogique ne se laisse pas aisément bafouer et continue à être âprement débattu en regard des premiers, ce qui explique la variabilité et l'instabilité dans les législations nationales. Toutes d'ailleurs n'ont pas encore statué sur ces sujets très controversés.

La gestation pour autrui pose quant à elle des problèmes additionnels qu'il faut aussi maîtriser. Le soupçon d'adultère, déjà présent dans les dons de gamètes, intervient avec plus de vraisemblance dans cette option. Pour déjouer cette crainte, des gestatrices peuvent avoir recours au symbolique et, par une sorte de travail discursif de cartographie, de body map (Teman 2010), visant à distinguer le circuit sexuel de celui de la gestation, elles pensent réussir à déjouer les accusations d'adultère entre elles et les donneurs de sperme. Si, de plus, la gestatrice est apparentée à l'un ou l'autre parent d'intention, s'ajoute à l'adultère la suspicion

23. "First-degree intergenerational collaborations need special scrutiny in view of the increased risk of undermining autonomous choice " (cf. : www.eshre.eu/ESHRE/English/Press-Room/PressReleases/2011-Press-Releases/IMAR/page.aspx/1206). 
d'inceste, et d'autres stratégies doivent alors être mises concurremment à l'œuvre. Une femme californienne, qui portait l'enfant de son frère et de sa belle-sœur, utilisait quant à elle l'argument qu'elle avait subi une ligature de trompes et que l'œuf, étant fécondé avant l'implantation, le sperme de son frère n'étant plus sexuellement actif, il n'y avait donc pas de possible soupçon d'inceste entre eux (Thompson 2005) ${ }^{24}$. Par ailleurs, la maternité de substitution crée, chez certains protagonistes, un autre type de relation vue comme incestueuse et pourtant inconnue jusque-là : celle entre enfants non consanguins mais portés par la même femme. Une seule des informatrices britanniques de Jeanette Edwards (2004) remarque que ces enfants-là ne devraient pas être autorisés à avoir des rapports sexuels ou à s'épouser. Ces façons de penser ne sont pas sans rappeler les interdits d'inceste qui s'instaurent entre personnes ayant été allaitées par une même nourrice. Le partage de substances vitales ou de fluides corporels crée, ici ou ailleurs, aujourd'hui comme jadis, de l'identité.

En outre, toutes les configurations familiales ne tombent pas sous le coup des mêmes interdits. À propos de la parenté adoptive, on note que dans les législations canadienne et française ${ }^{25}$, les empêchements de mariage avec les membres proches de la parenté d'origine continuent de s'appliquer même en cas d'adoption plénière, ce qui atteste de la force et du caractère indélébile du critère de la nature dans notre parenté occidentale. Encore faut-il, pour ne pas enfreindre la loi involontairement, connaître l'identité des parents de naissance, ce qui est impossible, en France du moins, en cas d'adoption d'enfant né sous X ou de ressortissants étrangers en provenance de certains pays, comme la Chine qui refuse de fournir tout renseignement précis sur l'identité des géniteurs. De plus, pour ces enfants adoptés s'ajoutent aux interdits liés à la naissance ceux créés par la parenté adoptive. Au Québec ${ }^{26}$, l'étendue de ces derniers peut varier en ligne collatérale en fonction du nombre d'années de vie familiale commune entre les personnes concernées, la législation tenant compte du critère de la "parenté du quotidien" (Weber 2005).

24. Cf., à ce sujet, le cas de Jane, mère porteuse canadienne qui avait elle aussi subi une ligature de trompes (Delaisi de Parseval \& Collard 2007).

25. L'article 577 du Code civil du Québec (1991, c. 64, a. 577 ; 2002, c. 6, a. 32) dit ceci : "L'adoption confere à l'adopté une filiation qui se substitue à sa filiation d'origine et, sous réserve des empêchements de mariage ou d'union civile, l'adopté cesse d'appartenir à sa famille d'origine, à moins que le tribunal ait décidé de ne pas rompre le lien préexistant de filiation ». En France, l'article 356 du Code civil dispose : "L'adoption confere à l'enfant une filiation qui se substitue à sa filiation d'origine: l'adopté cesse d'appartenir à sa famille par le sang, sous réserve des prohibitions au mariage visées aux articles 161 à 164 ».

26. Comme en témoigne, pour le Québec, l'article 578 du Code civil du Québec : "L’adoption fait naître les mêmes droits et obligations que la filiation par le sang. Toutefois, le tribunal .../... 
En ce qui concerne les familles recomposées, les empêchements de mariage entre demi-germains et donc demi-consanguins s'appliquent complètement. En revanche, il n'existe au Québec aucune loi interdisant le mariage entre beaux-parents et beaux-enfants alors que de telles interdictions existent en France. Dans l'un et l'autre pays, le mariage est possible entre " quasi » frères et sœurs. Remarquons toutefois que, dans un contexte de violence, ces situations relèvent de l'inceste. En effet, le code pénal du Québec qualifie comme criminels les viols et abus sexuels commis par des proches parents biologiques ou sociaux sur des mineurs dont ils ont la charge. En France, une telle législation pénale a été tentée mais jusqu'à présent, elle n'a pas abouti. En effet, la loi de février 2010 inscrivait l'inceste en tant que délit dans le Code pénal ${ }^{27}$ et stipulait " que les viols et les agressions sexuelles sont qualifiés d'incestueux lorsqu'ils sont commis au sein de la famille sur la personne d'un mineur par un ascendant, un frère, une sœur ou par toute autre personne, y compris s'il s'agit d'un concubin d'un membre de la famille, ayant sur la victime une autorité de droit ou de fait » (art. 22-31-1 du CP). Mais, par décision $\mathrm{du}$ Conseil constitutionnel de septembre 2011 (décision n²011-163 QPC), la loi a été abrogée au prétexte que la définition de la "proximité " familiale n'était pas assez précise. Ces hésitations expriment, à n’en pas douter, la difficulté qu'éprouvent nos sociétés devant ces configurations familiales nouvelles, à décider qui est apparenté ou pas, qui peut être considéré comme un proche ou un lointain parent. Mais, et c'est là que l'étude et l'analyse de la pratique de la parenté sont importantes à observer pour autant que l'on constate que des prohibitions additionnelles, culturellement intériorisées, se sont de façon informelle mises en place dans ces familles recomposées ${ }^{28}$. Les membres de ces fratries ainsi réunies

[Suite de la note 26] peut, suivant les circonstances, permettre un mariage ou une union civile en ligne collatérale entre l'adopté et un membre de sa famille d'adoption" (1991, c. 64, a. $578 ; 2002$, c. 6, a. 33). En France, la loi semble plus sévère et une telle directive ne peut s'appliquer qu'en cas d'adoption simple. L'article 366 du Code civil dispose, en effet, que "Le lien de parenté résultant de l'adoption s'étend aux enfants de l'adopté. Le mariage est prohibé : 1) entre l'adoptant, l'adopté et ses descendants ; 2) entre l'adopté et le conjoint de l'adoptant ; réciproquement entre l'adoptant et le conjoint de l'adopté ; 3) entre les enfants adoptifs du même individu ; 4) entre l'adopté et les enfants de l'adoptant. Néanmoins les prohibitions au mariage portées aux $3^{\circ}$ et $4^{\circ}$ ci-dessus peuvent être levées par dispense du président de la République, s'il y a des causes graves. La prohibition de mariage portée au $2^{\circ}$ ci-dessus ne peut être levée dans les mêmes conditions que lorsque la personne qui a créé l'alliance est décédée ».

27. Rappelons, qu'en France, jusqu’à cette loi de 2010 et depuis la Révolution de 1789, le législateur avait traité l'inceste comme un tabou moral qui relève de l'intime, du personnel et non comme un délit à inscrire dans la loi pénale.

28. On lira, à ce propos, l'article de Sylvie Kerviel, paru dans Le Monde des 6 et 7 février 2011, et intitulé "Mon quasi-frère, mon amour : quand les familles recomposées flirtent avec l'inceste ». 
s'interdisent d'avoir des relations sexuelles ou de nouer des unions entre quasi-frères et sœurs surtout quand ils ont été élevés ensemble et ont partagé une même intimité (Martial 2003). Quoi qu’il en soit, dans les familles adoptives ou recomposées, l'étude de ces interdits sexuels légaux ou culturels inédits montre bien que l'on ne saurait parler d'une "génétisation " ou d'une "biologisation" actuelle de la parenté particulièrement renforcée en Occident (Fine \& Martial 2010). Or, on constate, au contraire, avec la révélation de ces façons de faire que, malgré l'impact de nos connaissances scientifiques, la parenté sociale s'exprime toujours avec force.

$\mathrm{Si}$, malgré tous les efforts psychiques ou symboliques pour "courtcircuiter" les échanges de substances entre les partenaires familiaux, l'inceste resurgit là où on l'attend le moins, on comprend, dès lors, que cette crainte affleure sans cesse dans l'esprit de ceux et celles qui ne connaissent pas leurs origines parce qu'adoptés ou nés par dons anonymes de gamètes ${ }^{29}$.

\section{L'inceste accidentel}

L'adoption plénière, nationale ou internationale, avec secret des origines de l'enfant, et surtout l'insémination artificielle avec donneurs anonymes de gamètes, réveillent les fantasmes voire les peurs ancestrales d'un inceste accidentel, notamment entre entiers ou demi-germains génétiques ${ }^{30}$. Une peur telle, qu'elle peut être exacerbée à partir du simple soupçon de ressemblance :

«Ce qui me préoccupe aussi, c'est que je peux en toute légalité épouser mon frère sans le savoir ou, en toute ignorance, croiser mon père dans la rue. Et ça me fait toujours bizarre quand quelqu'un me dit: "Mais tu ressembles trop à Untel”. Ça n’a pas du tout le même effet que pour quelqu'un qui connaît ses deux parents génétiques " (Mehl $2008: 243)$.

La ressemblance ici active l'imaginaire de l'inceste accidentel, alors que, dans d'autres circonstances, elle conforte et solidifie le lien entre parents et enfant né par PMA. C'est ce qu'il advient lorsque des personnes, qui ne sont pas au courant du mode de naissance d'un enfant, disent de celui-ci qu'il ressemble à ses parents d'intention (Fortier 2009). Ces situations de

29. Cf., entre autres témoignages, l'ouvrage d'Arthur Kermalvezen \& Béatrice de Dinéchin (2008). 30. «Une femme passée du don anonyme au don direct témoigne: "J’ai eu peur que mes ovules fécondés et réimplantés dans le corps d'une femme inconnue ne donnent une grossesse, puis un enfant que j’ai imaginé être un garçon. Ce garçon, devenu adulte, pourrait par hasard rencontrer une de mes filles, en tomber amoureux sans savoir, que ce pourrait être sa sœur... Ceci ne doit pas arriver, je préfere vraiment donner à quelqu'un que je connais" " (Flis-Trèves 1992 : 106-107). 
méconnaissance font que se crée, autour de la PMA, une sorte de halo d'incertitudes parentales, différent selon qu'on est donneur, receveur ou rejeton. Surtout qu'apparemment, les risques réels d'inceste entre personnes nées d'un même don de sperme anonyme ne sont pas nuls, en particulier dans les pays où ces dons sont peu ou mal réglementés. Aux États-Unis où c'est le cas, le Donor Sibling Registry ${ }^{31}$ a été capable d'établir qu'un donneur avait contribué à la naissance de cent cinquante enfants et que des dons ayant donné lieu à plus de cinquante naissances n'étaient pas rares $^{32}$. Une donneuse australienne d'ovocytes a contribué, à elle seule, à la naissance de dix-neuf enfants ${ }^{33}$. On comprend, à la lecture de tels chiffres, pourquoi l'inceste accidentel concerne d'abord la progéniture, et donc la relation horizontale de "germanité ", plutôt que le lien vertical entre géniteur et descendance. Curieusement, ce ne sont pas toujours les médecins des cliniques de fertilité qui sont les plus concernés par les dangers génétiques de la proche consanguinité ${ }^{34}$ - encore que nombre d'entre eux le soient et en tiennent compte dans leur pratique -, mais, bien plus, les enfants conçus avec tiers donneurs et leurs parents. Voici, par exemple, comment s'exprime un informateur californien - État où le nombre de dons n'est pas réglementé - à propos des dons anonymes :

"Je pense que tout cela a créé un problème majeur et que c'est actuellement hors contrôle. Et maintenant... comment allons-nous reprendre les rênes de tout cela? ». Sa femme Sarah acquiesce et ajoute : «Et comment peut-on retracer combien de fois quelqu'un a fait des dons et où sont ces enfants? Et vous savez, nous ne voulons pas que nos enfants puissent aller au collège avec leur demi-frère et tomber amoureux sans même savoir qu'il s'agit d'un parent!» (notre traduction).

31. Le Donor Sibling Registry appelé encore le "Kramer» du nom de la personne qui est à l'origine de ce registre en ligne, volontaire, permettant aux donneurs de sperme ou d'ovules et aux enfants qui en sont nés de déposer leur ADN, et ainsi de communiquer entre eux et d'échanger des informations médicales (cf. Le Devoir 2011). Pour la France, on peut consulter le site internet de l'Association pour la procréation anonyme (http://www.pmanonyme.asso.fr).

32. "One Sperm Donor, 150 Offsprings ", New York Times (5 septembre 2011).

33. «IVF: Australian woman sets new record for egg donation» (http://tinyurl.com/Royston2011-06-01).

34. Comme en témoignent les propos de ce médecin chef d'une clinique de fertilité canadienne, interviewé par Chantal Collard en 2011. Celui-ci utilise des données anthropologiques qui ne sont pas fausses en elles-mêmes, car, comme on le sait, le mariage entre cousins existe bien dans plusieurs cultures à travers le monde, mais sans jamais spécifier le degré de parenté dans ses propos pour minimiser les risques génétiques d'inceste en PMA: "Well, it's much better in the old days when nobody told anybody anything. And then there were no problems. It's just like incest. Incest in some societies is perfectly acceptable. But we make a big deal of it and so people get upset when there is incest in the family in our culture. But there's some cultures where incest is everyday business where there's no stigma and no long term disadvantages anywhere or any injuries that result because it's culturally acceptable. But in our society it's different. There are some very vocal people that, you know, think they have a right to know their genetic parents and so on ». 
Naturellement, ce danger d'un inceste accidentel s'impose avec plus de force dans les cas de dons d'embryons qui, en France, sont comme tous les autres dons procréatifs, anonymes ${ }^{35}$. En Californie, toutes les personnes interrogées dans le cadre du programme Snowflakes d'adoption ouverte d'embryons, qu'elles aient donné ou reçu des embryons, ont souligné que l'inceste accidentel était, pour elles, une préoccupation majeure et que c'était en grande partie pour cette raison qu'elles s'étaient orientées vers un processus d'adoption ouverte (Collard \& Kashmeri 2009, 2011). Tous les parents de cette enquête comptaient informer leurs enfants, au plus tard à l'adolescence, pour éviter les risques d'inceste et d'intermariage, et la grande majorité d'entre eux laissait ouverte la possibilité, pour leurs enfants, de développer par la suite avec leurs autres "germains » des relations de proximité, indépendamment ou non de leurs parents respectifs. Au demeurant, une minorité d'entre eux avait déjà établi des contacts suivis avec les autres familles donneuses ou receveuses (Collard \& Kashmeri 2011). Comme on le pressent, ces relations peuvent déboucher sur un type de familles (post)composées par germanité, différent de celles (re)composées par l'alliance et le divorce. Encore qu'il serait nécessaire, pour mieux définir et nommer ce phénomène, d'en savoir un peu plus sur les modalités et le sens de ces liens de parenté qui se tissent entre ces familles. Marylin Strathern (1999) le souligne d'ailleurs : la connaissance des liens biologiques est une règle constitutive de la parenté, préalable et indispensable à la reconnaissance sociale de celle-ci. Une fois connue la nature de la relation, on ne peut l'ignorer ni revenir en arrière et faire comme si elle n'existait pas, mais il n'en demeure pas moins que tous ces frères et sœurs "de gènes" et leurs géniteurs ne développeront pas tous de véritables relations de parenté même s'ils éviteront de commettre l'inceste. Ainsi que le dit la sagesse populaire: trop de parenté nuit à la parenté ${ }^{36}$. Dans les cas d'enfants nés par don de gamètes ou d'embryons d'un géniteur et/ou d'une génitrice communs, on constate que lorsque les contacts se poursuivent, ils n'entraînent pas la totalité de la fratrie lorsque celle-ci est trop étendue, mais qu'ils sont sélectifs et s'arrêtent à un maximum gérable qui correspond en gros à une fratrie «normale ", à une famille «normale ». Au-delà, on n'est plus dans la parenté mais dans l'«humanité" où tous les hommes sont frères! Le corps, pensé comme nécessaire dénominateur commun de la parenté,

35. Cf., à ce propos, l'article de Geneviève Delaisi de Parseval et Pauline Tiberghien, paru dans le journal Libération du 18 septembre 2004, qui qualifient cet anonymat de "bombe à retardement ". 36. Cette expression résume bien les représentations de la parenté en milieu traditionnel, en France rurale tout particulièrement (cf. Zonabend 2000). 
n'est en effet pas suffisant. Il faut que le processus d' "emparentement» soit amplifié par d'autres relations de proximité d'âge, de voisinage, de langue et d'histoire partagés. Toutefois, pour tous ces parents potentiels demeure, comme en suspens entre eux, cet interdit de l'inceste et d'exogamie. Aussi devons-nous insister: comment contourner ou gérer le risque d'inceste lorsqu'il y a anonymat des dons de gamètes?

Parmi les États qui persistent dans le choix de dons anonymes, certains ont opté, afin de rendre le risque génétique acceptable, pour une réglementation légale limitant le nombre de dons que peut effectuer un même individu, et le nombre d'enfants issus de ces dons: ainsi l'utilisation du sperme d'un donneur est limitée à la naissance de dix enfants en France et les ovules d'une même donneuse ne peuvent bénéficier qu'à deux couples receveurs depuis la loi de bioéthique de 2004, en Belgique à six, aux Pays-Bas à vingt-cinq. En Autriche, un homme ne peut donner son sperme que dans une seule clinique et pas plus de trois couples peuvent l'utiliser. Aux États-Unis, l'American Society for Reproductive Medecine recommande de limiter les dons à vingt-cinq naissances pour 800000 habitants, mais la plupart des banques de sperme ne suivent pas ces recommandations.

Une autre stratégie, mise en place par les receveurs ou les donneurs, consiste à faire appel à ces sites internet dont il a été question plus haut, pour retracer, en déposant en ligne sa formule ADN, les donneurs de gamètes et les enfants issus des mêmes dons que soi. Cette méthode est très populaire en Amérique du Nord en l'absence de toute régulation légale.

On peut aussi militer pour la levée de l'anonymat au nom des droits de l'enfant à connaître ses origines (Delaisi de Parseval 2008; Théry 2010). Ces revendications ont abouti, en Grande-Bretagne, en Norvège ou en Suisse, à la levée de l'anonymat du donneur lorsque l'enfant a atteint quatorze ans ou plus, comme pour l'adoption. Ailleurs, d'autres procédés ont été mis en place qui font appel à ces nouveaux " généalogistes-gérantsdu-secret ${ }^{37}$ que sont les registres des cliniques de fertilité ou des commissions étatiques. En France, le Conseil national d'accès aux origines personnelles (CNAOP), créé par la loi de janvier 2002, a pour tâche de faciliter l'accès des receveurs, des enfants et des donneurs, s'ils le veulent, à leur identification réciproque. Au Canada, si les donneurs ont consenti

37. Rappelons, en effet, que ces organismes administratifs ont tous pouvoirs de décision en matière de "mémoire généalogique" de ceux qui les sollicitent. Ainsi, la CNAOP a décidé que, même après le délai de cinquante ans qui permet d'avoir accès aux archives des personnes, "l'identité d'un parent de naissance qui a demandé que celle-ci soit préservée de son vivant aussi bien qu'après son décès est donc effectivement incommunicable " (circulaire DGP/SIAF/ AACR/2010/011, du 27 juillet 2010). 
à ce que leur identité soit divulguée, des mécanismes sont en place dans les législations fédérales et provinciales pour ce faire. Par ailleurs, en l'absence de consentement des donneurs sur leur identité, la loi fédérale de $2004{ }^{38}$ permet de fournir certains renseignements génétiques non identifiants aux requérants, aux fins d'éviter en amont les dangers d'inceste :

"Sur demande écrite de deux personnes qui sont fondées à croire qu'au moins l'une d'elles est issue d'une technique de procréation assistée au moyen du matériel reproductif humain ou d'un embryon in vitro d'un donneur, l'Agence les informe du fait qu'elle a ou non en sa possession des renseignements qui indiquent qu'elles sont génétiquement parentes et, le cas échéant, de la nature du lien de parenté» (Article 18.4).

Accessoirement, en différant la connaissance des géniteurs à l'adolescence, on tente de maintenir le plus longtemps possible le principe généalogique classique qui pose (voire, impose toujours) : " pas plus de deux parents ». Mais, un jour ou l'autre, sans que se développe forcément de la parenté, sous l'influence du nombre de plus en plus important de naissances assistées et de cette crainte de plus en plus forte d'un inceste accidentel, l'anonymat ne pourra se maintenir. Faut-il dès lors penser que le modèle généalogique, si présent à travers l'histoire de l'Occident et dont David Schneider (1984) a prétendu qu'on l'avait indûment appliqué au reste du monde, est encore opérationnel et adéquat? Ou faut-il affirmer - ou préconiser - que coexiste, du moins dans ces cas-là, un double modèle généalogique, d'un côté purement génétique et biologique, de l'autre purement social?

Ce double modèle généalogique, qui peu ou prou se met en place actuellement, encore complexifié par la présence plus ou moins marginale de ces frères et sœurs "de gènes ", indique clairement qu'en bout de ligne le tour est joué : la prohibition de l'inceste remet de l'ordre généalogique et de la sexualité dans la "parenté sans sexualité » puisque ces pères et mères, ces frères et sœurs, ne pourront ni avoir de rapports sexuels entre eux (ni avec leurs cogéniteurs par IAD ou don de gamètes ou d'embryons) ni procréer ou s'allier. Bien évidemment, les interdits d'inceste inscrits dans la parenté sociale continueront de s'appliquer. Tout se passe comme si réapparaissait, une fois satisfaite par des façons détournées la possibilité 
d'« entrer en parenté » et de "faire » des enfants, une exigence irrésistible et irréductible - et qui explique à elle seule la survie de la parenté -, celle du corps et de la sexualité par le biais de l'inceste.

Université Concordia

Département de sociologie et d'anthropologie, Montréal (Canada)

chantal.collard@concordia.ca

Laboratoire d'anthropologie sociale, Paris

zonabend@ehess.fr

MOTS CLÉS/KEYWORDS : nouvelles parentés/new forms of kinship - inceste/incest - sexualitél sexuality - filiation/filiation - germanité/siblingship.

\section{BIBLIOGRAPHIE}

Aristote

2002 De la génération des animaux. Éd. par Pierre Louis. Paris, Les Belles Lettres ( Collection des Universités de France. Série grecque»152).

Bamford, Sandra C.

2004 "Conceiving Relatedness : NonSubstantial Relations among the Kamea of Papua New Guinea ", Journal of the Royal Anthropological Institute 10 (2) : 287-306.

2007 Biology Unmoored. Melanesian Reflections on Life and Biotechnology. Berkeley, University of California Press.

Bamford, Sandra C. \& James Leach, eds 2009 Kinship and Beyond. The Genealogical Model Reconsidered. New York, Berghahn.

Barry, Laurent

2008 La Parenté.

Paris, Gallimard

(«Folio Essais» 498).

2013 La Prohibition de l'inceste.

Paris, L'Harmattan-Pepper [À paraitre].
Bestard, Joan

2009 «Knowing and Relating: Kinship Assisted Reproductive Technologies and the New Genetics ", in Jeanette Edwards \& Carles Salazar, eds, European Kinship in the Age of Biotechnology... : 19-28.

Boltanski, Luc

2004 La Condition foetale. Une sociologie de l'engendrement et de l'avortement.

Paris, Gallimard ("NRF essais»).

Bonte, Pierre, Enric Porqueres i Gené \& Jérôme Wilgaux, eds

2011 L'Argument de la filiation. Aux fondements des sociétés européennes et méditerranéennes. Paris, Éd. de la MsH ( Méditerranée-Sud»).

Boswell, John

1993 Au bon cour des inconnus.

Les enfants abandonnés de l'Antiquité

à la Renaissance. Trad. de l'anglais

par Pierre-Emmanuel Dauzat. Paris, Gallimard ("Bibliothèque des histoires»). 
Brunet, Laurence
2011 «Des usages protéiformes de la nature : essai de relecture du droit français de la filiation ", in Pierre Bonte, Enric Porqueres i Gené \& Jérome Wilgaux, eds, L'Argument de la filiation... : 285-323.

\section{Cadoret, Anne}

2009 «Parenté et familles homoparentales: le grand chahut ", in Enric Porqueres

i Gené, ed., Défis contemporains de la parenté... : 151-170.

\section{Cahn, Naomi}

2009 "Accidental Incest: Drawing the Line - or the Curtain ? - for Reproductive Technology ", Harvard Journal of Law \& Gender 32 : 59-107.

\section{Cai, Hua}

1997 Une société sans père ni mari,

les $\mathrm{Na}$ de Chine. Paris, Presses

universitaires de France

(«Ethnologies»).

\section{Carsten, Janet}

1995 "The Substance of Kinship and the Heat of the Hearth : Feeding, Personhood and Relatedness among Malays in Pulau Langkawi ", American Ethnologist 22 (2) : 223-241.

2000 "Introduction ", in Janet Carsten, ed., Cultures of Relatedness. New Approaches to the Study of Kinship. Cambridge, Cambridge University Press : 1-36.

2011 «Substance and Relationality: Blood in Contexts ", Annual Review of Anthropology 40 : 19-35.

\section{Collard, Chantal \& Shireen Kashmeri} 2009 " "De embriones congelados a siempre familias" : ética del parentesco y ética de la vida en la circulación de embriones entre las parejas donantes y las adoptantes en el programa Snowflakes ", Revista de antropologia social 18 : 43-65.

2011 «Embryo Adoption : Emergent Forms of Siblingship among the Snowflakes Families ", American Ethnologist 38 (2): 307-322.
Corbier, Mireille, ed.

1999 Adoption et fosterage.

Paris, De Boccard

(«De l'archéologie à l'histoire»).

Delaisi de Parseval, Geneviève

2008 Famille à tout prix.

Paris, Le Seuil

(«La Couleur des idées »).

Delaisi de Parseval, Geneviève

\& Chantal Collard

2007 «La gestation pour autrui : un bricolage des représentations de la paternité et de la maternité euro-américaines ", L'Homme 183 : 29-54.

\section{D'Onofrio, Salvatore}

2004 L'Esprit de la parenté. Europe et horizon chrétien. Paris, Éd. de la MsH.

\section{Edwards, Jeanette}

2004 «Incorporating Incest :

Gamete, Body and Relation in Assisted Conception ", Journal of the Royal Anthropological Institute 10 (4) : 755-774.

2009 "La vie sociale du sang et des gènes", in Enric Porqueres i Gené, ed., Défis contemporains de la parenté... : 303-326.

Edwards, Jeanette \& Carles Salazar, eds 2009 European Kinship in the Age of Biotechnology. New York, Berghahn Books.

Fine, Agnès

1994 Parrains, marraines.

La parenté spirituelle en Europe.

Paris, Fayard.

Fine, Agnès \& Agnès Martial

2010 "Vers une naturalisation de la filiation", Genèses 78 : 121-134.

Fine, Agnès \& Claire Neirinck, eds 2000 Parents de sang, parents adoptifs. Approches juridiques et anthropologiques de l'adoption: France, Europe, USA, Canada. Paris, LGDJ ("Droit et société. Série anthropologique»). 
Finkler, Kaja

2001 "The Kin in the Gene:

The Medicalization of Family and Kinship in American Society ", Current Anthropology $42(2): 235-263$.

\section{Flis-Trèves, Muriel}

1992 "Une femme, un homme, un couple demande un enfant ", Les Cahiers de l'IPPC 13: 103-108.

\section{Fortier, Corinne}

2001 «Le lait, le sperme, le dos:

et le sang ? Représentations physiologiques de la filiation et de la parenté de lait en islam malékite et dans la société maure de Mauritanie », Cahiers d'études africaines $161: 97-138$.

2005 «Le don de sperme et le don d'ovocyte ou "trois font un" : sexualité, inceste et procréation ", in Jacques Galinier, Patrice Bidou \& Bernard Juillerat, eds, Anthropologie et psychanalyse. Regards croisés. Paris, Éd. de l'EHESs ("Cahiers de L'Homme » 37) : 59-80.

2009 «Quand la ressemblance fait la parenté ", in Enric Porqueres i Gené, ed., Défis contemporains de la parenté... : 251-276.

\section{Frydman, René}

2008 «OGM : origine génétiquement modifiée ", in René Frydman \& Muriel Flis-Trèves, eds, Origines de la vie, vertiges des origines. Paris, Presses universitaires de France : 223-224.

\section{Godelier, Maurice}

2004 Les Métamorphoses de la parenté.

Paris, Fayard.

Héran, François

2009 Figures de la parenté. Une histoire critique de la raison structurale. Paris, Presses universitaires de France («Sociologies»).

\section{Héritier, Françoise}

1994 Les Deux Socurs et leur mère. Anthropologie de l'inceste. Paris, Odile Jacob.
Héritier, Françoise \& Élisabeth Copet-

Rougier, eds

1995 La Parenté spirituelle.

Paris, Éd. des Archives contemporaines

(«Ordres sociaux»).

Howell, Signe

2003 « Kinning: The Creation

of Life Trajectories in Transnational

Adoptive Families ", Journal

of the Royal Anthropological Institute 9 (3):

465-484.

Hutchinson, Sharon E.

1996 Nuer Dilemmas Coping

with Money, War, and the State.

Berkeley, University of California Press.

Juillerat, Bernard

2005 «Plaisir et réalité : la notion

de "cargo" revisitée ", in Jacques Galinier,

Patrice Bidou \& Bernard Juillerat, eds,

Anthropologie et psychanalyse.

Regards croisés. Paris, Éd. de l'EHESS

("Cahiers de l'Homme» 37) : 81-97.

Kermalvezen, Arthur

\& Béatrice de Dinéchin

2008 Né de spermatozoïde inconnu.

Paris, Presses de la Renaissance.

Leblic, Isabelle, ed.

2004 De l'adoption. Des pratiques de filiation différentes. Clermont-Ferrand, Presses universitaires Blaise Pascal («Anthropologie »).

Lévi-Strauss, Claude

1976 [1955] Tristes Tropiques.

Paris, Plon («Terre humaine»).

McKinnon, Susan

2005 «On Kinship and Marriage :

A Critique of the Genetic and Gender

Calculus of Evolutionary Psychology ", in Susan McKinnon \& Sydel Silverman, eds, Complexities. Beyond Nature

and Nurture. Chicago,

University of Chicago Press : 106-131. 
MacLaury, Robert E.

1991 "Prototypes revisited ", Annual

Review of Anthropology 20 : 55-74.

Malinowski, Bronislaw

1929 The Sexual Life of Savages in North-

Western Melanesia. An Ethnographic Account of Courtship, Marriage and Family Life among the Natives of the Trobriand Islands, British New Guinea. London, Routledge \& Sons.

\section{Martial, Agnès}

2003 S'apparenter. Ethnologie des liens de familles recomposées. Paris, Éd. de la MsH ("Ethnologie de la France» 25).

Mehl, Dominique

2008 Enfants du don.

Procréation médicalement assistée :

parents et enfants témoignent.

Paris, Robert Laffont

(«Le monde comme il va»).

Mejia Quijano, Claudia, Marc Germond

\& François Ansermet, eds

2006 Parentalité stérile et procréation médicalement assistée. Le dégel du devenir.

Ramonville-Saint-Agne, Erès

( La vie de l'enfant»).

Menget, Patrick

1988 « Note sur l'adoption chez les Txicào du Brésil central ", Anthropologie et sociétés $12(2)$ : 63-72.

\section{Moisseeff, Marika}

2002 «L'utérus, une boîte de Pandore d'où peuvent s'échapper mille démons ", Soins. Psychiatrie 221 : 24-26.

\section{Porqueres i Gené, Enric}

2009 «Individu et personne à la lumière des nouveaux contextes de la parenté ", in Enric Porqueres i Gené, ed., Défis contemporains de la parenté... : 225-250.

Porqueres i Gené, Enric, ed.

2009 Défis contemporains de la parenté. Paris, Éd. de l'EHESS (“Cas de figure»7).
Pullman, Bertrand

2010 Mille et une façons de faire les enfants. La révolution des méthodes de procréation. Paris, Calmann-Levy.

Sahlins, Marshall

2011 "What Kinship Is ", Journal of the Royal Anthropological Institute 17 (1) : 2 -19 (Part one) ; 17 (2) : 227-242 (Part two). 2012 "Birth is the Metaphor ", Journal of the Royal Anthropological Institute 18 (3): 673-677.

Schneider, David M.

1980 [1968] American Kinship.

A Cultural Account.

Chicago, University of Chicago Press.

1984 A Critique of the Study of Kinship.

Ann Arbor, University of Michigan Press.

Shapiro, Warren

2008 «What Human Kinship is Primarily About: Toward a Critique of the New Kinship Studies ", Social Anthropology 16 (2) : 137-153.

2012 «Extensionism and the Nature of Kinship ", Journal of the Royal Antropological Institute 18 (1) : 191-193.

Sissa, Julia

1991 «Philosophies du genre: Platon, Aristote et la différence des sexes", in Georges Duby \& Michèle Perrot, eds, Histoire des femmes en Occident, 1. L'Antiquité. Paris, Plon : 65-99.

Strathern, Marylin

1992 After Nature. English Kinship in the Late Twentieth Century. CambridgeNew York, Cambridge University Press 1995 «Displacing Knowledge :

Technology and the Consequences for Kinship ", in Fay D. Ginsburg \& Rayna Rapp, eds, Conceiving the New World Order. The Global Politics of Reproduction. Berkeley, University of California Press : 346-364.

1999 Property, Substance, and Effect. Anthropological Essays on Persons and Things. London, Athlone Press. 


\section{Taylor, Anne-Christine}

2000 «Le sexe de la proie: représentations jivaro du lien de parenté ", L'Homme 154-155 : 309-334.

Teman, Elly

2010 "The Body Map and Intended Mothers and Maternal Intentions", in Birthing a Mother. The Surrogate Body and the Pregnant Self. Berkeley, University of California Press.

Théry, Irène

2010 Des humains comme les autres.

Bioéthique, anonymat et genre du don.

Paris, Éd. de l'EHESS (“Cas de figure»14).

Thompson, Charis

2005 Making Parents. The Ontological

Choreography of Reproductive Technologies.

Cambridge, MrT Press.

Vernier, Bernard

2009 La Prohibition de l'inceste. Critique de Françoise Héritier. Paris, L'Harmattan.

Viveiros de Castro, Eduardo

2004a «Perspectival Anthropology and the Method of Controlled Equivocation", Tipitì 2:3-22. 2004b «Le don et le donné :

trois nano-essais sur la parenté et la magie", Ethnographiques.org 6

(http://www.ethnographiques.org/2004/ Viveiros-de-Castro).

2009 "The Gift and the Given : Three Nano-Essays on Kinship and Magic ", in Sandra Bamford \& James Leach, eds, Kinship and Beyond... : 237-268.

\section{Weber, Florence}

2005 Le Sang, le nom, le quotidien.

Une sociologie de la parenté pratique.

Paris, Aux lieux d'être

("Mondes contemporains»).

Weiner, Annette, B.

1987 The Trobrianders of Papua

New Guinea. New York,

Holt, Rinehart \& Winston.

\section{Zonabend, Françoise}

2000 "Les maîtres de parenté : une femme de mémoire en Basse-Normandie ", L'Homme 154-155 : 505-524.

2007 «Adopter des sœurs : construction de la parenté et mémoire des origines", L'Homme 183 : 9-28. 
Chantal Collard \& Françoise Zonabend, Parenté sans sexualité : le paradigme occidental en question. - Les multiples façons d'accéder à la parenté par l'intermédiaire des avancées de la médecine procréative, ont toutes pour dénominateur commun l'absence de recours à l'union sexuelle de l'homme et (ou) de la femme qui en sont les bénéficiaires. Dès lors, celles-ci remettent en cause les grands piliers conceptuels sur lesquels reposent classiquement le système occidental de la parenté. D'où l'interrogation qui parcourt ce texte: peut-on encore parler de parenté, avec ces nouvelles façons de se fabriquer des parents? L'exploration des récentes recherches tant anthropologiques que juridiques ou psychologiques menées dans ce domaine, tant dans des sociétés exotiques qu'occidentales, anciennes ou contemporaines, montrent toutes qu'une sexualité et donc une corporéité ressurgit, par le biais de l'inceste, au sein de ces nouvelles façons de "faire parenté ", les réinscrivant, en quelque sorte, dans la continuité.
Chantal Collard \& Françoise Zonabend, Kinship without Sexuality:The Western Paradigm Called in Question. - What is common to the many methods for attaining kinship through advances in reproductive medicine is the absence of a sexual relation between the beneficiaries, whether men or women. This calls in question the major conceptual pillars that classically buttress the Western kinship system. In effect, can we still talk about kinship given these new methods for making parents? A review of recent studies in anthropology, law or psychology on this topic, in exotic as well as Western societies, ancient or contemporary, discovers that, in all of them, sexuality and, as a consequence, a corporeality re-emerge through incest in all these new methods for "making kingship ", thus placing them in a continuity. 